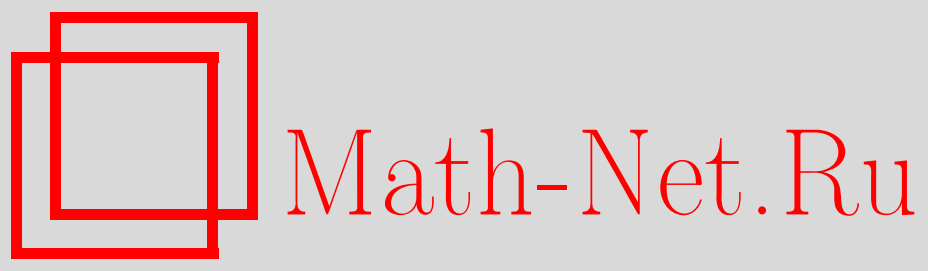

И. М. Дектярев, Аналоги существенно особых точек для последовательностей полиномиальных отображений, Функи. анализ и его прил., 2001, том 35, выпуск 4, 26-31

DOI: https://doi.org/10.4213/faa270

Использование Общероссийского математического портала MathNet.Ru подразумевает, что вы прочитали и согласны с пользовательским соглашением

http://www.mathnet.ru/rus/agreement

Параметры загрузки:

IP : 54.174 .149 .18

26 апреля 2023 г., 14:51:23

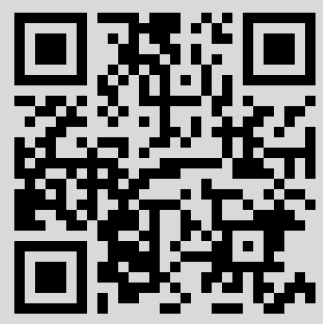




\title{
Аналоги существенно особых точек для последовательностей полиномиальных отображений*
}

\author{
(C) 2001. И. М. ДЕКтяРев
}

Рассмотрим последовательность отображений $F_{k}: \mathbb{C}^{2} \rightarrow \mathbb{C}^{2} \subset \mathbb{C P}^{2}$, задаваемых формулами $F_{k}\left(z_{1}, z_{2}\right)=\left(1: z_{1}^{k}: z_{2}^{k}\right)$, где $\left(w_{0}: w_{1}: w_{2}\right)-$ однородные координаты в пространстве $\mathbb{C P}^{2}$. Очевидно, что для любого положительного $\varepsilon$ и любой точки $w^{(0)}$ пространства $\mathbb{C P}^{2}$ (включая расположенные на «бесконечно удаленной» прямой $\left.\left\{w_{0}=0\right\}\right)$ в шаре радиуса $\sqrt{2}+\varepsilon$ пространства $\mathbb{C}^{2}$ найдется такая последовательность точек $z^{(1)}, \ldots, z^{(k)}, \ldots$, что последовательность $F_{1}\left(z^{(1)}\right), \ldots, F_{k}\left(z^{(k)}\right), \ldots$ при $k \rightarrow \infty$ сходится к $w^{(0)}$. С другой стороны, если все точки $z^{(1)}, \ldots, z^{(k)}, \ldots$ принадлежат шару радиуса $\sqrt{2}-2 \varepsilon$, то хотя бы одна координата любой из этих точек не превосходит $1-\varepsilon$, и поэтому предельными для последовательности $\left\{F_{k}\left(z^{(k)}\right)\right\}$ могут быть только те точки $w^{(0)}$, у которых хотя бы одна из координат равна нулю. Более того, для любой точки $z^{(0)}=\left(z_{1}^{(0)}, z_{2}^{(0)}\right)$, удовлетворяющей условиям $\left|z_{1}^{(0)}\right|=\left|z_{2}^{(0)}\right|=1$ (и только для таких точек), последовательность $\left\{z^{(k)}\right\}$ можно выбрать так, чтобы она сходилась к $z^{(0)}$.

В настоящей статье мы покажем, что эти наблюдения отражают некоторые общие закономерности поведения последовательностей полиномиальных отображений.

Пусть $F: X \rightarrow Y$ - голоморфное отображение $n$-мерных комплексных многообразий, и пусть на многообразии $Y$ задана форма объема $\omega$. Будем предполагать, что интеграл $\int_{X} F^{*} \omega$, определяющий индуцированный объем многообразия $X$, конечен. Обозначим его через $\operatorname{vol}(X, F)$. Для области $U \subset X$ определим относительный объем $\operatorname{relvol}(U, F)$ как отношение $\operatorname{vol}(U, F) / \operatorname{vol}(X, F)$.

Если отображение $F: \mathbb{C}^{n} \rightarrow \mathbb{C}^{n} \subset \mathbb{C P}^{n}$ задается формулой $F\left(z_{1}, \ldots, z_{n}\right)=$ $\left(1: f_{1}: \cdots: f_{n}\right)$, где $f_{1}, \ldots, f_{n}-$ полиномы, у которых однородные компоненты старших степеней не имеют общих нулей, за исключением тривиального, то будем называть его полиномиальным отображением общего вида. Если $F-$ полиномиальное отображение общего вида, а форма $\omega-$ нормированный элемент объема метрики Фубини-Штуди на пространстве $\mathbb{C} \mathbb{P}^{n}$, то величина $\operatorname{vol}\left(\mathbb{C}^{n}, F\right)$ равна топологической степени $\operatorname{deg}(F)$ отображения $F$, которая в данном случае совпадает с произведением степеней полиномов $f_{1}, \ldots, f_{n}$.

Целью работы является доказательство следующих теорем.

Теорема 1. Пусть $F_{1}, \ldots, F_{k}, \ldots$ - последовательность полиномиальных отображений общего вида. Обозначим через $d_{k}$ наименьшую из степеней полиномов $f_{1}^{(k)}, \ldots, f_{n}^{(k)}$, задающих отображение $F_{k}$. Если для некоторого шара $B(r, a)$ пространства $\mathbb{C}^{n}$ радиуса $r$ с центром в точке а последовательность

*Работа выполнена при поддержке Российского фонда фундаментальных исследований, грант 99-01-00115. 
произведений $\left\{d_{k} \operatorname{relvol}\left(B(r, a), F_{k}\right)\right\}$ стремится $\kappa$ бесконечности, то для любой точки $w^{(0)} \in \mathbb{C P}^{n}$ и любого положительного в найдется такая последовательность точек $z^{(1)}, \ldots, z^{(k)}, \ldots$, принадлежащих шару $B(r+\varepsilon, a)$, ито последовательность $F_{1}\left(z^{(1)}\right), \ldots, F_{k}\left(z^{(k)}\right), \ldots$ при $k \rightarrow \infty$ стремится $\kappa w^{(0)}$.

Следующая теорема утверждает, что при выполнении определенных условий точка пространства $\mathbb{C}^{n}$ как бы является существенно особой относительно последовательности отображений.

Теорема 2. Пусть $F_{1}, \ldots, F_{k}, \ldots$ - последовательность таких же отображений, как в теореме 1. Если для некоторой точки а пространства $\mathbb{C}^{n}$ найдется такая последовательность иаров $\left\{B\left(r_{k}, a\right)\right\}$, радиусы которых стремятся к нулю, что последовательность произведений $\left\{d_{k} \operatorname{relvol}\left(B\left(r_{k}, a\right), F_{k}\right)\right\}$ ограничена снизу положительной константой, то для любой точки $w^{(0)} \in$ $\mathbb{C P}^{n}$ найдется такая последовательность точек $z^{(1)}, \ldots, z^{(k)}, \ldots$, сходящзаяся к точке а, ито последовательность $F_{1}\left(z^{(1)}\right), \ldots, F_{k}\left(z^{(k)}\right), \ldots$ при $k \rightarrow \infty$ стремится $\kappa w^{(0)}$.

Доказательство будет опираться на следующую лемму.

Лемма 1. Пусть F- полиномиальное отображение общего вида, и пусть $d_{F}$ - наименьшая из степеней полиномов $f_{1}, \ldots, f_{n}$, задающих отображение $F$. Пусть образ некоторого иара $B(R, a)$ пространства $\mathbb{C}^{n}$ не пересекается с каким-нибудь геодезическим шаром радиуса $\rho$ пространства $\mathbb{C P}^{n}$. Тогда для каждого $r, 0<r<R$, выполнено следующее соотношение:

$$
\operatorname{relvol}(B(r, a), F) d_{F} \rho^{2 n-2}\left(\frac{R^{2}}{1+R^{2}} \ln \frac{R^{2}}{r^{2}}-\ln \frac{1+R^{2}}{1+r^{2}}\right) \leqslant c,
$$

где константа с зависит только от размерности.

Следующая лемма нами использоваться не будет, но она представляет самостоятельный интерес, а по доказательству и по сути тесно примыкает к предыдущей лемме.

Лемма 2. Пусть $F$ - полиномиальное отображение общзего вида, и пусть $d_{F}$ - наименьшая из степеней полиномов $f_{1}, \ldots, f_{n}$, задающих отображение $F$. Пусть образ некоторого шара $B(R, a)$ пространства $\mathbb{C}^{n}$ полностью содержится в каком-нибудь шаре радиуса $\rho_{1}$ (здесь радиус измеряется в метрике пространства $\left.\mathbb{C}^{n}\right)$. Тогда для каждого $r, 0<r<R$, величинь $r, R, \rho_{1}$ u $\operatorname{relvol}(B(r, a), F)$ удовлетворяют следующему соотношению:

$$
\operatorname{relvol}(B(r, a), F) d_{F}\left(\frac{R^{2}}{1+R^{2}} \ln \frac{R^{2}}{r^{2}}-\ln \frac{1+R^{2}}{1+r^{2}}\right) \leqslant c \ln \left(1+\rho_{1}^{2}\right),
$$

где константа с зависит только от размерности.

ДоКАЗАТЕЛЬСтвО ЛЕммы 1. Нормируем метрику Фубини-Штуди на пространстве $\mathbb{C P}^{n}$ так, чтобы объем всего $\mathbb{C P}^{n}$ равнялся единице, и обозначим через $\omega$ форму, задающую элемент объема в этой метрике. Пусть $\varphi-$ положительная дважды непрерывно дифференцируемая функция на пространстве $\mathbb{C}^{n}$, носитель которой содержится в шаре $B(R, a)$. Тогда для величины $\operatorname{relvol}(B(r, a), F)$ получаем соотношение $m \operatorname{relvol}(B(r, a), F) \operatorname{deg}(F) \leqslant \int_{\mathbb{C}^{n}} \varphi F^{*} \omega$, где $m-$ нижняя грань значений функции $\varphi$ на шаре $B(r, a)$. С другой стороны, в работе [1] доказано существование на $\mathbb{C} \mathbb{P}^{n}$ такого семейства функций $\gamma_{w}(y)$, что при $y \neq w$ эти 
функции являются гладкими и выполнено соотношение $d d^{c}\left(\gamma_{w}(y) \mu\right)=\omega$. Через $\mu$ здесь обозначена $(n-1)$-я внешняя степень кэлеровой формы, ассоциированной с нормированной метрикой Фубини-Штуди. Если $w^{(0)}$ - центр того геодезического шара, с которым не пересекается $F(B(R, a))$, то, учитывая, что носитель функции $\varphi$ содержится в шаре $B(R, a)$, имеем

$$
\int_{\mathbb{C}^{n}} \varphi F^{*} \omega=\int_{B(R, a)} \varphi F^{*} d d^{c}\left(\gamma_{w^{(0)}}(y) \mu\right)=\int_{B(R, a)} \gamma_{w^{(0)}}(F(z)) d d^{c} \varphi \wedge F^{*} \mu .
$$

Возьмем в качестве $\varphi$ функцию $\Theta\left(\|z-a\|^{2}\right)$, где $\Theta(s)=(4 \pi)^{-1} \int_{s}^{R^{2}} \theta(x) \ln (x / s) d x$, а функция $\theta(x)$ на отрезке $\left[0, r^{2}\right]$ определяется как $\alpha x+\beta$, а на отрезке $\left[r^{2}, R^{2}-\varepsilon\right]$ - как $1 /(1+x)^{2}$. При $x \geqslant R^{2}$ функция $\theta(x)$ полагается равной нулю, а на отрезке $\left[R^{2}-\varepsilon, R^{2}\right]$ она определяется как линейная функция с тем, чтобы оказаться непрерывной в концах этого отрезка. Малой величиной $\varepsilon$ мы распорядимся позднее. Коэффициенты $\alpha$ и $\beta$ определяются из условия непрерывности функции $\theta$ в точке $r^{2}$ (т. е. $\left.\alpha r^{2}+\beta=1 /\left(1+r^{2}\right)^{2}\right)$ и отсутствия особенностей у функции $\Theta(s)$ при $s=0$. Последнее условие обеспечивается равенством $\int_{0}^{R^{2}} \theta(x) d x=0$. Действительно, интегрируя по частям выражение для $\Theta(s)$, в этом случае получаем

$$
\Theta(s)=-\frac{1}{4 \pi} \int_{s}^{R^{2}}\left(\frac{1}{x} \int_{0}^{x} \theta(t) d t\right) d x=\frac{1}{4 \pi} \int_{s}^{R^{2}}\left(\frac{1}{x} \int_{x}^{R^{2}} \theta(t) d t\right) d x .
$$

На интервале $\left(0, r^{2}\right)$ функция $\Theta(s)$ будет равна

$$
-\frac{1}{4 \pi}\left(\frac{\alpha}{4}\left(r^{4}-s^{2}\right)+\beta\left(r^{2}-s\right)+\int_{r^{2}}^{R^{2}}\left(\frac{1}{x} \int_{0}^{x} \theta(t) d t\right) d x\right) .
$$

Функция $\Theta(s)$, ее первая производная $-(4 \pi)^{-1} s^{-1} \int_{s}^{R^{2}} \theta(x) d x$ и вторая производная $(4 \pi)^{-1} s^{-2} \int_{s}^{R^{2}} \theta(x) d x+(4 \pi)^{-1} s^{-1} \theta(s)$ непрерывны на $[0, \infty)$ и удовлетворяют уравнению $s \Theta^{\prime \prime}(s)+\Theta^{\prime}(s)=(4 \pi)^{-1} \theta(s)$. Кроме того, на интервале $\left(0, R^{2}\right)$ сама функция положительна, а ее первая производная отрицательна. Минимальное значение $m$ функции $\Theta(s)$ на отрезке $\left[0, r^{2}\right]$ равно

$$
\Theta\left(r^{2}\right)=\frac{1}{4 \pi} \int_{r^{2}}^{R^{2}}\left(\frac{1}{x} \int_{x}^{R^{2}} \theta(t) d t\right) d x
$$

и при подходящем выборе $\varepsilon$ может быть сделано сколь угодно близким к величине $\frac{1}{4 \pi}\left(\frac{R^{2}}{1+R^{2}} \ln \frac{R^{2}}{r^{2}}-\ln \frac{1+R^{2}}{1+r^{2}}\right)$.

Покажем, что если функция $\psi(s)$ удовлетворяет неравенствам $\psi^{\prime}(s) \geqslant 0$ и $s \psi^{\prime \prime}(s)+\psi^{\prime}(s) \geqslant 0$, то форма $d d^{c} \psi\left(\|z-a\|^{2}\right)$ положительна. Действительно, эта форма равна

$$
\psi^{\prime \prime}\left(\|z-a\|^{2}\right) d\|z-a\|^{2} \wedge d^{c}\|z-a\|^{2}+\psi^{\prime}\left(\|z-a\|^{2}\right) d d^{c}\|z-a\|^{2},
$$

и, чтобы убедиться в ее положительности в точке $z^{(0)}$, достаточно выбрать систему координат так, чтобы все координаты точки $z^{(0)}-a$, кроме первой, равнялись нулю. 
Нужными свойствами обладает функция $(4 \pi)^{-1} \ln (1+s)-\Theta(s)$, а это означает, что

$$
\begin{aligned}
& \int_{B(R, a)} \gamma_{w^{(0)}}(F(z)) d d^{c} \Theta\left(\|z-a\|^{2}\right) \wedge F^{*} \mu \\
& \leqslant \frac{1}{4 \pi} \int_{B(R, a)} \gamma_{w^{(0)}}(F(z)) d d^{c} \ln \left(1+\|z-a\|^{2}\right) \wedge F^{*} \mu .
\end{aligned}
$$

Из приведенного в [1] явного вида функции $\gamma_{w}(y)$ следует, что вне геодезического шара радиуса $\rho$ с центром в $w^{(0)}$ ее значения не превосходят величины $c \rho^{-2 n+2}$, где константа $c$ зависит только от размерности, и нам достаточно доказать, что

$$
\frac{1}{4 \pi} \int_{\mathbb{C}^{n}} d d^{c} \ln \left(1+\|z-a\|^{2}\right) \wedge F^{*} \mu=\frac{\operatorname{deg}(F)}{d_{F}} .
$$

Для этого воспользуемся леммой 4.2 работы [2]. Там рассматривается набор $\left\{F_{k}\right\}$ рациональных отображений пространства $\mathbb{C} \mathbb{P}^{n}$ в пространства $\mathbb{C P}^{m_{k}}$, и для каждого такого отображения через $I_{k}$ обозначается множество неопределенности, а через $I-$ объединение $\bigcup_{i=1}^{n} I_{k}$. Через $\omega_{k}$ обозначена кэлерова форма, ассоциированная с нормированной метрикой Фубини-Штуди на соответствующем пространстве $\mathbb{C P}^{m_{k}}$, а через $F_{k}^{-1}(H)$ для $H \subset \mathbb{C P}^{m_{k}}$ обозначено множество $\pi_{1}\left(\pi_{2}^{-1}(H)\right)$, где $\pi_{1}$ и $\pi_{2}-$ соответствующие проекции графика отображения $F_{k}$. Лемма утверждает, что интеграл $\int_{\mathbb{C P}^{n} \backslash I} F_{1}^{*} \omega_{1} \wedge \cdots \wedge F_{n}^{*} \omega_{n}$ равен количеству точек множества $\bigcap_{i=1}^{n} F_{k}^{-1}\left(H_{k}\right) \backslash I$ для «общего» набора гиперплоскостей $\left\{H_{k} \subset \mathbb{C P}^{m_{k}}\right\}$.

Мы зададим отображение $F_{1}$ формулой $F_{1}(z)=z-a$, а в качестве отображений $F_{2}=\cdots=F_{n}$ возьмем изучаемое нами отображение $F$. Будем считать эти отображения продолженными до рациональных отображений $\mathbb{C} \mathbb{P}^{n} \rightarrow \mathbb{C} \mathbb{P}^{n}$. Отображение $F_{1}$ голоморфно, а множество неопределенности отображения $F$ является пересечением «бесконечно удаленной» гиперплоскости с множеством общих нулей однородных составляющих степени $p$ полиномов, задающих это отображение. Здесь $p$ - это наибольшая из степеней полиномов из рассматриваемой системы. В частности, если все полиномы имеют степень $p$, то для полиномиальных отображений общего вида множество $I$ пусто. Подынтегральное выражение в цитируемой лемме совпадает с нужным нам, а область интегрирования отличается на множество меры нуль. Если гиперплоскость $H$ задать соотношением $\alpha_{0} w_{0}+\cdots+\alpha_{n} w_{n}=0$, а отображение $F$ в однородных координатах записать в виде $F\left(z_{0}, \ldots, z_{n}\right)=\left(z_{0}^{p}: \tilde{f}_{1}: \cdots: \tilde{f}_{n}\right)$, то множество $F^{-1}(H)$ совпадает с множеством нетривиальных нулей полинома $\alpha_{0} z_{0}^{p}+\alpha_{1} \tilde{f}_{1}+\cdots+\alpha_{n} \tilde{f}_{n}$.

Предположим сначала, что все полиномы, образующие отображение $F$, имеют степень $p$. Поскольку $F$ - полиномиальное отображение общего вида, у полиномов $\tilde{f}_{1}, \ldots, \tilde{f}_{n}$ нет нетривиальных общих корней в бесконечно удаленной гиперплоскости $\left\{z_{0}=0\right\}$, а множество нулей, расположенных в $\mathbb{C}^{n}$, будучи аналитическим множеством в компактной части этого пространства, конечно. Значит, по теореме Бертини для «общего» набора гиперплоскостей $\left\{H_{k}\right\}$ система уравнений, определяющих множество $\bigcap_{i=1}^{n} F_{k}^{-1}\left(H_{k}\right)$, имеет лишь изолированные решения первого порядка, а по теореме Безу количество точек в множестве решений равно произведению степеней этих уравнений, т.е. $p^{n-1}$ (напомним, что степень уравнения, определяющего множество $F_{1}^{-1}\left(H_{1}\right)$, равна единице). А это, в свою очередь, равно $\operatorname{deg}(F) / d_{F}$. 
Пусть теперь не все полиномы имеют степень $p$. Поскольку множество неопределенности содержится в гиперплоскости $\left\{z_{0}=0\right\}$, в цитируемой лемме необходимо подсчитать количество корней, для которых $z_{0} \neq 0$. Положим для определенности $z_{0}=1$. Здесь опять применима теорема Бертини, и соответствующая система уравнений имеет лишь изолированные решения первого порядка. Будем считать, что полиномы пронумерованы так, что их степени не возрастают. Подвергая уравнения, соответствующие гиперплоскостям $H_{k}$ для $k=$ $2, \ldots, n$, преобразованиям Жордана-Гаусса, можно добиться, что они примут вид $f_{k-1}+\alpha_{k} f_{n}=\beta_{k}$ и получившаяся система (вместе с первым уравнением) будет по-прежнему иметь лишь изолированные решения первого порядка. Эти решения устойчивы в том смысле, что при малых изменениях коэффициентов они не исчезают, а лишь перемещаются на малое расстояние. Сколь угодно малым изменением коэффициентов без изменения степеней полиномов можно добиться того, что у получившейся системы будут отсутствовать «бесконечно удаленные» решения и, значит, можно будет применить теорему Безу. С учетом выбранной нумерации полиномов получаем нужное утверждение.

ДОКАЗАТЕЛЬСТВО ЛЕМмЫ 2 Проводится точно так же, но вместо функций $\gamma_{w}(y)$ из [1] воспользуемся функцией $\psi(y)=(4 \pi)^{-1} \ln \left(1+\|y\|^{2}\right)$. Она также удовлетворяет нужному уравнению $d d^{c}(\psi(y) \mu)=\omega$, но вместо ее оценки вне геодезического шара радиуса $\rho$ с центром в $w^{(0)}$ следует воспользоваться неравенством для ее значений внутри шара радиуса $\rho_{1}$ пространства $\mathbb{C}^{n}$.

ДоКАЗАТЕЛЬСТВО ТЕОРЕмЫ 1 . Положим $R=r+\varepsilon$ и обозначим через $\rho_{k}$ точную верхнюю грань радиусов тех геодезических шаров с центром в точке $w^{(0)}$, которые не пересекаются с $F_{k}(B(R, a))$. Если таких шаров нет, то положим $\rho_{k}$ равным $1 / k$. Таким образом, для каждого $k$ в шаре $B(R, a)$ найдется такая точка $z^{(k)}$, что $F_{k}\left(z^{(k)}\right)$ отстоит от точки $w^{(0)}$ не более, чем, например, на $2 \rho_{k}$. С другой стороны, из условий теоремы, согласно лемме 1, следует, что последовательность $\left\{\rho_{k}\right\}$ стремится к нулю и потому последовательность $\left\{z^{(k)}\right\}$ является требуемой.

ДокАЗАТЕЛЬСтвО ТЕОРЕмЫ 2. Выберем на интервале $(0,1)$ положительную функцию $R(x)$, полагая ее равной $(\ln (1 / x))^{-1 / 3}$. Тогда при $x \rightarrow 0$ величина $R(x)$ стремится к нулю, в то время как функция $\frac{R(x)^{2}}{1+R(x)^{2}} \ln \frac{R(x)^{2}}{x^{2}}-\ln \frac{1+R(x)^{2}}{1+r(x)^{2}}$ стремится к бесконечности. Для каждого $k$ положим $R_{k}=R\left(r_{k}\right)$. Далее так же, как и при доказательстве теоремы 1 , но используя для каждого $k$ свои $R_{k}$ и $r_{k}$, построим последовательности $\left\{\rho_{k}\right\}$ и $\left\{z^{(k)}\right\}$. По построению последовательность $\left\{R_{k}\right\}$ стремится к нулю, а значит, $\left\{z^{(k)}\right\}$ стремится к $a$. Учитывая условия теоремы 2 и применяя лемму 1 к отображениям $F_{k}$ и числам $R_{k}$ и $r_{k}$, получаем, что $\left\{\rho_{k}\right\}$ стремится к нулю, т.е. $\left\{F_{k}\left(z^{(k)}\right)\right\}$ сходится к $w^{(0)}$.

ЗАмЕЧАНИЕ 1. С очевидными изменениями все проведенные выше рассуждения проходят и для одномерного случая. Здесь даже не надо требовать полиномиальности отображений. Достаточно, чтобы они задавались функциями, мероморфными в круге радиуса $r+\varepsilon$. При этом величину $\operatorname{relvol}(B(r, a), F) d_{F}$ следует заменить на площадь круга в индуцированной метрике. Однако в одномерном случае известны более сильные результаты. Если дополнение к объединению образов круга радиуса $r+\varepsilon$ содержит три различные точки, то семейство отображений будет нормальным. Применяя принадлежащий Ф. Марти критерий нормальности 
(см., например, [3, с. 238]), получим противоречие с условиями, наложенными на последовательность отображений. Получается аналог теоремы Пикара, в то время как у нас - лишь аналог теоремы Вейерштрасса.

ЗАмЕЧАниЕ 2. Естественно возникает вопрос: в какой мере полиномиальность отображений необходима в многомерном случае? Автору представляется, что ответ не должен получаться просто. Одним из препятствий является, например, следующий факт. Пусть $G=\left(g_{1}, g_{2}\right): \mathbb{C}^{2} \rightarrow \mathbb{C}^{2}-$ голоморфное отображение, при котором дополнение к образу содержит открытое множество (см. примеры ФатуБибербаха). Тогда последовательность отображений $F_{k}=\left(g_{1}\left(z_{1}^{k}, z_{2}^{k}\right), g_{2}\left(z_{1}^{k}, z_{2}^{k}\right)\right)$, казалось бы, всем хороша, однако при подходящем выборе отображения $G$ дополнение к объединению образов может содержать бесконечное число областей, каждая из которых биголоморфно эквивалентна пространству $\mathbb{C}^{2}$.

\section{ЛИТЕРАТУРА}

1. Dektyarev I. M. Multivariable value distribution theory. Application to holomorphic curves. J. Math. Sci., 92, No. 2, 3685-3711 (1998).

2. Russakouskii A., Shiffman B. Value distribution for sequences of rational mappings and complex dynamics, Indiana Univ. Math. J., 46, No. 3, 897-932 (1997).

3. Хейман У. К. Мероморфные функции. Мир, М., 1966.

Владимирский государственный педагогический университет
Поступило в редакцию 29 мая 2000 г. 